DR. MARCELLO ESPOSITO (Orcid ID : 0000-0003-2034-9018)

DR. ALFREDO BERARDELLI (Orcid ID : 0000-0003-3598-3142)

DR. ROBERTO ERRO (Orcid ID : 0000-0002-7800-7419)

DR. TOMMASO SCHIRINZI (Orcid ID : 0000-0002-2517-6278)

DR. GIOVANNI COSSU (Orcid ID : 0000-0002-9129-2584)

DR. FRANCESCA MORGANTE (Orcid ID : 0000-0002-9834-3639)

DR. MAURIZIO ZIBETTI (Orcid ID : 0000-0002-2939-343X)

PROF. GIOVANNI DEFAZIO (Orcid ID : 0000-0001-5950-3110)

Article type : Original Article

\title{
Spread of dystonia in patients with idiopathic adult-onset laryngeal dystonia.
}

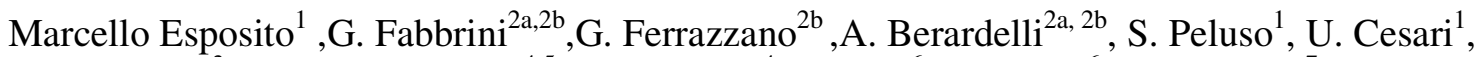

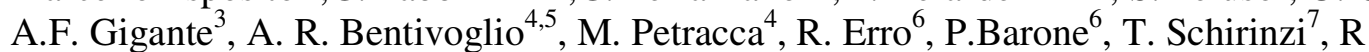
Eleopra $^{8}$, L. Avanzino ${ }^{9}$, M. Romano ${ }^{10}$, C.L. Scaglione ${ }^{11}$, G.Cossu $^{12}$, F. Morgante ${ }^{13}$, B.

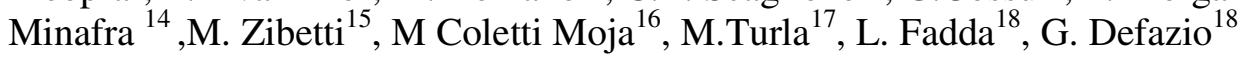

${ }^{1}$ Department of Neurosciences, Reproductive Science and Odontostomatology, Federico II University of Naples, Naples, Italy

${ }^{2 a}$ Department of Neurology and Psychiatry, Sapienza University of Rome

${ }^{2 \mathrm{~b}}$ IRCCS Neuromed Pozzilli, Italy

${ }^{3}$ Department of Basic Science, Neuroscience and Sense Organs, Aldo Moro University of Bari, 70124 Bari, Italy

${ }^{4}$ Movement Disorders Unit, Center for Parkinson's Disease and Extrapyramidal Disorders, Institute of

Neurology, Catholic University, Rome, Italy

${ }^{5}$ Don Carlo Gnocchi Onlus Foundation, Milan Italy

${ }^{6}$ Center for Neurodegenerative Diseases (CEMAND), Neuroscience Section, University of Salerno, Salerno, Italy

${ }^{7}$ Department of Systems Medicine, University of Rome "Tor Vergata", Rome, Italy

${ }^{8}$ IRCCS Foundation C. Besta Neurological Institute, Milan, Italy

${ }^{9}$ Section of Human Physiology, Department of Experimental Medicine, University of Genoa, Genoa, Italy

${ }^{10}$ Neurology Unit, Villa Sofia Hospital, Palermo, Italy

${ }^{11}$ IRCCS Institute of Neurological Sciences, Bologna, Italy

12 Department of Neurology, AOB “G. Brotzu' General Hospital, Cagliari, Italy

${ }^{13}$ Department of Neuroscience, University of Messina, Messina, Italy

${ }^{14}$ Parkinson's Disease and Movement Disorders Unit, C. Mondino National Neurological Institute, IRCCS Pavia, Italy

${ }^{15}$ Department of Neuroscience “Rita Levi Montalcini'”, University of Turin, Turin, Italy

${ }^{16}$ Neurology Unit, Umberto I Hospital, Turin, Italy

This article has been accepted for publication and undergone full peer review but has not been through the copyediting, typesetting, pagination and proofreading process, which may lead to differences between this version and the Version of Record. Please cite this article as doi: 10.1111/ene.13731

This article is protected by copyright. All rights reserved 
${ }^{17}$ Neurology Unit, Valle Camonica Hospital, Brescia, Italy
${ }^{18}$ Department of Medical Sciences and Public Health, University of Cagliari, Cagliari, Italy.

Running title: Spread of dystonia with laryngeal onset

Key words: laryngeal dystonia, adult focal dystonia, spread

Supplemental material online: none

Conflict of Interest: none

Funding for the study: none

\section{Corresponding Author:}

Marcello Esposito

Department of Neurosciences, Reproductive Science and Odontostomatology, Federico II

University of Naples

Via Pansini, 5 Naples, Italy

email: marcelloesposito@live.it

\section{Abstract}

Introduction: Adult onset laryngeal dystonia (LD) can be isolated or can be associated to dystonia in other body parts. Combined forms can be segmental at the onset or can result from dystonia spread to or from larynx. Aim of this study is to identify main clinical and demographic features of adult onset idiopathic LD in an Italian population with special focus on dystonia spread. Methods: Data were obtained from the Italian Dystonia Registry (IDR) produced by 37 Italian Institutions. Clinical and demographic data of 71 patients with idiopathic adult-onset LD were extracted from a pool of 1131 subjects included in the IDR. Results: 50 of 71 patients presented a laryngeal focal onset, remaining subjects had onset in other body regions and later laryngeal spread. The two groups did not show significant differences of demographic features. $32 \%$ of patients with laryngeal onset reported spread to contiguous body regions afterwards and in most of cases (12 of 16 subjects) dystonia started to spread within one year from the onset. LD patients who remained focal and those who had dystonia spread did not show other differences. Discussion: data from IDR show that 
dystonic patients with focal laryngeal onset will present spread in almost one third of cases. Spread from larynx occurs early and it is directed to contiguous body regions showing similarities with clinical progression of blepharospasm. This study gives a new accurate description of LD phenomenology that may contribute to improve the comprehension of dystonia pathophysiology.

\section{Introduction}

Adult-onset laryngeal dystonia (LD) is a relatively rare form of action-induced dystonia that predominates in women and has a peak age of onset in the $5^{\text {th }}$ to 6 th decade [1]. The most frequent form is adductor LD that is due to involuntary adduction of the vocal folds during speech and produces a strained/strangled speaking pattern. The less common abductor LD is caused by overactivity of the abductor muscles during speech, with excessive opening of the vocal folds and prolonged breathy voice breaks or whispering speaking pattern [2]. Botulinum toxin is very effective to treat $\mathrm{LD}$ and recurring injections in laryngeal muscles can produce persistent benefits over time [3].

Over the disease course, LD may remain focal or spread to near body regions. LD may also develop after dystonia emerged in a body part other than the larynx [2]. Although the relative risk of spread of dystonia, important for clinical prognosis and approach, has been well characterized in blepharospasm (BSP), cervical dystonia (CD), and upper limb dystonia (ULD) [4], the few studies dealing with spread of dystonia in patients with LD [5-8] provided inconsistent results probably because of methodologic flaws. These included the relatively small size of most examined sample (15 to 117 patients), and the recruitment of patients with secondary and/or juvenile-onset dystonia that may be characterized by a different disease progression [5-8].

This article is protected by copyright. All rights reserved 
In this study we cross-sectionally examined the clinical and demographic features related to onset site and spread of LD in a cohort of Italian patients with adult-onset idiopathic LD.

\section{Methods}

Data were obtained from the Italian Dystonia Registry (IDR) database (9). Thirty-seven Italian Institutions contributed to the database using a common clinical protocol. Eligibility for the IDR required a diagnosis of dystonia according to published criteria [10-12] and age at dystonia onset greater than 17 years (9). Both neurologists and otolaryngologists contributed to the diagnosis of LD (12). Assessment included a standardized historical data collection and clinical examination of all the body sites potentially affected by dystonia (9). For each affected body region, the year of dystonia onset was recorded. We could therefore retrace dystonia course retrospectively and focus on the spread from the referred clinical onset. All patients with age at onset $<26$ years were tested and proved negative for DYT1 mutation [2].

Data from 71 patients with idiopathic adult-onset LD (from 16 Institutions.) were extracted from a pool of 1131 consecutively enrolled patients with idiopathic adult-onset dystonia. All data from January 1, 2016 (IDR start) through September 15, 2017 were used and included information collected at the first visit and at follow up examinations when possible. In each movement disorder center data at first and follow up visits were recorded only by neurologists with expertise in dystonia in order to ensure accuracy in data collection.

We only analyzed data from the first visit if a participant had additional follow up visits. No patient was excluded because of insufficient/missing data.

All patients gave informed consent to participate in the study which was approved by the local ethical committee of each Institutions ("Federico II University of Naples, Sapienza University of Rome, Neuromed in Pozzilli, Aldo Moro University of Bari, Catholic 
University of Rome, University of Salerno, University of Rome "Tor Vergata", Besta Neurological Institute in Milan, University of Genoa, Villa Sofia Hospital in Palermo, IRCCS of Neurological Sciences of Bologna, , University of Messina, IRCCS “Mondino" in Pavia, University of Turin, "Umberto I" Hospital in Turin, "Valle Camonica" Hospital in Brescia, University of Cagliari)

Statistical analysis was made by the Stata 11.0 package (StataCorp LP, College Station, TX). Data were expressed as mean \pm standard deviation_(SD) unless otherwise indicated. Differences between groups were analyzed by Mann- Whitney's U test, chi-square test and Fisher test (two-tailed) as appropriate. In patients with focal laryngeal onset, spread of dystonia was estimated by Kaplan-Meier's survival curves using the time elapsed between LD onset and spread as the study time. Patients in whom spread never occurred were included in the survival functions for the duration of the observation, and their data were censored beyond that time. For all analyses, significance was set at the 0.05 level.

\section{Results}

Demographic and clinical data for the 71 study subjects with LD are reported in Table 1 (column 1). The sample was characterized by female preponderance (79\%), mean age at dystonia onset in the 6th decade, mean disease duration of 10 years, mean age at spread in the 6th decade and a high frequency of adductor LD (84\%). Patients with adductor LD were 61 ( $14 \mathrm{M}, 47 \mathrm{~F})$ and with abductor LD were 10 (1 M, 9 F). Female preponderance was more evident in the abductor group but sex difference is comparable between the two groups (Fisher exact test, $\mathrm{p}=0.68$ ).

This article is protected by copyright. All rights reserved 
Among the 71 patients, 50 with had focal laryngeal onset (Table 1, column 2) and 21 had focal onset in another body region and later laryngeal spread (Table 1, column 3). Women predominated in both groups even though their percentage was greater in the group with focal laryngeal onset $(43 / 50$ vs. $13 / 21, p=0.02)$. The other features did not significantly differ between the two groups even though there was a non significant trend for a lower age at dystonia onset in the 21 patients with focal onset in a body region other than the larynx $(\mathrm{p}=$ 0.1). In these 21 patients, onset of dystonia was in the cranial region (N.8 patients), in the cervical region (N.10 patients), and in the upper limb (N.3 patients), and LD manifested $13.5 \pm 14.5$ years after dystonia onset.

Among the 50 participants who reported focal laryngeal onset, $16(32 \%)$ later spread to a contiguous body region (the neck in 11 patients, the cranial region in 5) whereas 34 remained focal over 1 to 56 years of follow-up. Kaplan-Meier's survival model based on the time elapsed between LD onset and spread (Figure 1 ) showed that 12/ 16 patients manifested spread of dystonia within a year from LD onset whereas in 4 patients spread events occurred 1 to 5 years after LD onset. Mean age at spread in the 16 patients was $58.6 \pm 16.3$ years. Patients who spread and those who did not were similar for sex (15 women/1 man vs. 28 women/6 men, $\mathrm{p}=0.4)$ and age at $\mathrm{LD}$ onset $(57.9 \pm 15.8$ vs. $55.8 \pm 16.4, \mathrm{p}=0.33)$. In the 34 patients who did not spread, mean disease duration was significantly longer than the mean time required for spread $(10.8 \pm 11.1$ vs. $1 \pm 1.2$ years, $\mathrm{p}<0.0001)$. In $10 / 34 \mathrm{LD}$ patients who did not spread, however, disease duration was shorter ( 1 to 4 years) than the maximum time required for spread (5 years).

Overall, LD was part of a segmental dystonia in 37 patients (16 with focal laryngeal onset and 21 with focal onset elsewhere). In these 37 patients, LD was most frequently associated with CD $(21 / 37,57 \%)$ or cranial dystonia $(13 / 37,35 \%)$, less frequently with upper limb dystonia $(3 / 37,8 \%)$.

This article is protected by copyright. All rights reserved 


\section{Discussion}

We found spreading of dystonia to contiguous body regions in about one third of patients presenting with idiopathic adult-onset focal LD dystonia. In this group, spread occurred within the first year of disease in the majority of patients, and cervical and cranial muscles were the most frequent sites of progression. A preferential association of LD with $\mathrm{CD}$ and cranial dystonia was also observed in patients with LD as part of a segmental dystonia and focal onset elsewhere.

The $32 \%$ frequency of dystonia spread in the group of patients presenting with focal LD is higher than previously reported (6.7 to 19\%) [4-7]. The inclusion of patients with secondary and/or juvenile-onset dystonia in some previous series [5-8] might have determined this difference, as these groups are not comparable for etiology and disease progression [2]. In addition, previous studies used retrospective assessment of clinical records [5-8], whereas we employed standardized historical data collection and clinical examination that might have been more sensitive in detecting dystonia spread.

The rate of spread observed in this sample suggests that the first 5 years of history may be crucial for spread of LD. This differs from what has been reported in CD ad ULD but resembles the figure for the rate of spread that has been consistently observed in BSP $[4,7]$. The variable tendency to spread showed by different focal dystonias has been linked, at least in part, to variations in age at dystonia onset. It is worth noting that in our patients with focal LD onset, age at LD onset and age at spread are closer to age at onset and age at spread of idiopathic adult-onset BSP than to the correspondent figures for CD and ULD $[1,4,7]$.

The more frequent association of LD with $\mathrm{CD}$ and cranial dystonia rather than with ULD might reflect a preferential diffusion of the functional abnormalities underlying dystonia over contiguous brain areas. Indeed, imaging studies have shown that phonation areas are closer 
to orofacial/neck areas than to upper limb areas in the primary motor cortex and in the basal ganglia, two structures thought to strongly contribute to the pathophysiology of dystonia [13].

Our study has limitations. This was not a population-based study and a referral bias cannot be ruled out. However, most demographic and clinical features resembled the general population of patients with LD [5-8]. The higher age at focal LD onset observed in the present series probably resulted from inclusion criteria strictly assessing patients with adultonset LD. The non significant trend for a lower age at dystonia onset in the patients with laryngeal dystonia and focal onset elsewhere (head, neck, or upper extremities) in comparison the patients with focal LD onset was probably due to the fact that CD and ULD usually develop at an earlier age than BSP and LD [1]. Owing to the relatively small size of the sample we could not stratify by adductor/abductor type of LD to check for differences in dystonia spread. Likewise, we did not examine family history of dystonia and its possible influence over spread. Although the mean duration of disease in patients remaining with focal $\mathrm{LD}$ at the end of the study period was much longer than the mean time required for spread, disease duration was shorter than the maximum time to spread in $10 / 34$ focal patients. This might have underestimated the true frequency of dystonia spread. Finally patients who spread were not regularly tested for THAP1 gene and, therefore, we could not completely exclude DYT6 dystonia in our cohort. [14]. We also did not test patients for GNAL mutations although a possible presentation of DYT 25 with isolated LD has been recently reported [15].

Despite the foregoing limitations, we are confident that information from this study is probably accurate and reflect the phenomenology of spread in adult-onset idiopathic LD. Our figures for rate and extent of spread may be relevant for design and implementation of future studies aiming to treat or modify disease progression in patients with idiopathic adult-onset LD.

This article is protected by copyright. All rights reserved 


\section{References}

1. O'Riordan S, Raymond D, Lynch T et al. Age at onset as a factor in determining the phenotype of primary torsion dystonia. Neurology. 2004;63:1423-1426.

2. Jinnah HA, Berardelli A, Comella $\mathrm{C}$ et al. Dystonia Coalition Investigators. The focal dystonias: current views and challenges for future research. Mov Disord. 2013;28:926-43.

3. Esposito M, Dubbioso R, Apisa P, Allocca R, Santoro L, Cesari U. Spasmodic dysphonia follow-up with videolaryngoscopy and voice spectrography during treatment with botulinum toxin. Neurol Sci. 2015 Sep;36(9):1679-82.

4. Abbruzzese G, Berardelli A, Girlanda P et al. Long-term assessment of the risk of spread in primary late-onset focal dystonia. J Neurol Neurosurg Psychiatry. 2008;79:392-396.

5. Bentivoglio AR, Elia AE, Filippini G, Valente EM, Fasano A, Albanese A. Clinical presentation and progression of sporadic and familial primary torsion dystonia in Italy. Adv Neurol 2004;94: 171-178.

6. Weiss EM, Hershey T, Karimi M et al. Relative risk of spread of symptoms among the focal onset primary dystonias. Mov Disord. 2006 Aug;21(8):117581. PubMed PMID: 16673404.

7. Svetel M, Pekmezović T, Jović J et al. Spread of primary dystonia in relation to initially affected region. J Neurol. 2007 Jul;254(7):879-83.

8. Blitzer A, Brin MF, Fahn S, Lovelace RE. Clinical and laboratory characteristics of focal laryngeal dystonia: study of 110 cases. Laryngoscope 1988;98(Pt. 1):636-640.

This article is protected by copyright. All rights reserved 
9. Defazio G, Esposito M, Abbruzzese G, et al. The Italian Dystonia Registry: rationale, design and preliminary findings. Neurol Sci. 2017 May;38:819-825

10. Albanese A, Bhatia K, Bressman SB et al. Phenomenology and classification of dystonia: a consensus update. Mov Disord. 2013;28:863-73.

11. Defazio G, Hallett M, Jinnah HA, Berardelli A. Development and validation of a clinical guideline for diagnosing blepharospasm. Neurology. 2013;81:236-40.

12. Ludlow C, Adler C, Berke G, et al. Research priorities in spasmodic dysphonia. Otolaryngol Head Neck Surg 2008;139:495-505.

13. Simonyan K, Horwitz B. Laryngeal Motor Cortex and Control of Speech in Humans. Neuroscientist 2011 17: 197

14. Djarmati A, Schneider SA, Lohmann K et al. Mutations in THAP1 (DYT6) and generalised dystonia with prominent spasmodic dysphonia: a genetic screening study. Lancet Neurol. 2009 May;8(5):447-52.

15. Putzel GG, Fuchs T, Battistella G, Rubien-Thomas E, Frucht SJ, Blitzer A,Ozelius LJ, Simonyan K. GNAL mutation in isolated laryngeal dystonia. Mov Disord. 2016 May;31(5):750-5. 


\section{ACKNOWLEDGMENT}

none

\section{AUTHOR DISCLOSURES}

All authors do not report disclosures

There was no funding for this study

Figure 1. Kaplan-Meier's survival analysis of spread of dystonia in patients with focal laryngeal dystonia at onset. Study time was represented by the time elapsed between dystonia onset and spread, and patients in whom spread never occurred were included in the survival function for the duration of the observation. The number of at-risk patients was 50 at time zero, 25 at 5 years, 16 at 10 years, 8 at 15 years.

This article is protected by copyright. All rights reserved 
TABLE 1. Demographic and clinical characteristics by laryngeal and non laryngeal site of dystonia onset .

\begin{tabular}{|c|c|c|c|c|}
\hline & All & $\begin{array}{l}\text { Focal } \\
\text { laryngeal onset }\end{array}$ & $\begin{array}{l}\text { Focal onset } \\
\text { elsewhere }\end{array}$ & $\mathrm{P}^{*}$ \\
\hline Number of patients & 71 & 50 & 21 & \\
\hline Mean age at study \pm SD (years) & $70.1 \pm 9.7$ & $68.1 \pm 11.7$ & $71.7 \pm 8.4$ & \\
\hline Number of women & 56 & 43 & 13 & 0.02 \\
\hline Mean years of education \pm SD & $9.7 \pm 4.4$ & $9.4 \pm 4.5$ & $10 \pm 4.4$ & 0.7 \\
\hline Mean age at dystonia onset \pm SD (years) & $54.9 \pm 18.2$ & $57.1 \pm 16.1$ & $52.8 \pm 13.7$ & 0.1 \\
\hline \multicolumn{5}{|l|}{ Type of laryngeal dystonia (N. patients) } \\
\hline Adductor & 61 & 43 & 18 & 1 \\
\hline Abductor & 10 & 7 & 3 & \\
\hline
\end{tabular}

*Data from column 2 vs. data from column 3

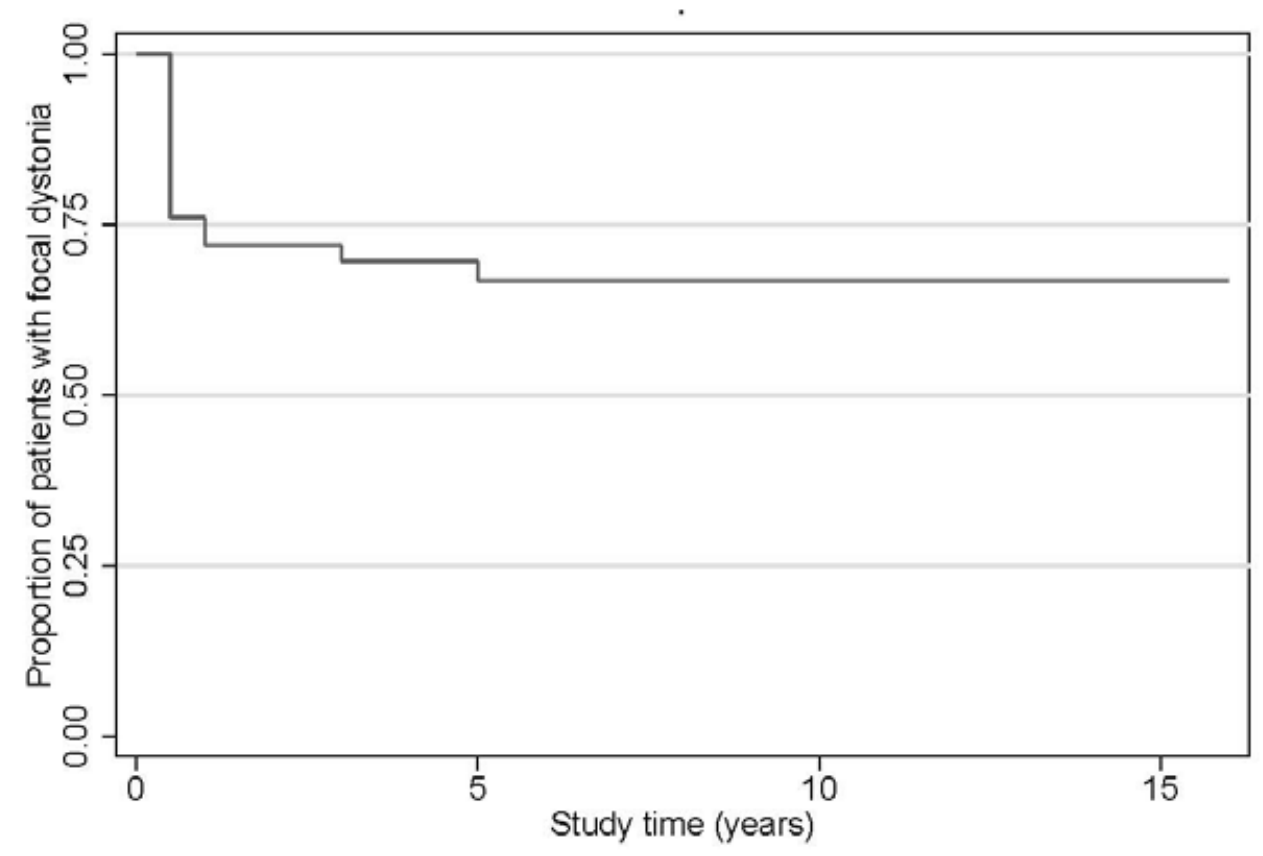

This article is protected by copyright. All rights reserved 